\title{
Performance analysis of rain attenuation at Ku-band in Malaysia
}

\begin{abstract}
Due to the lack of reliable analysis in Malaysia as tropical region, this study presents an analysis of experimental data compared against existing rain attenuation prediction models, namely the Dissanayake Allnut Haidara (DAH), and International Telecommunication Union rain ITU-R models, which have been used previously in satellite communication systems. Experimental data was measured at Universiti Putra Malaysia, Selangor, by retrieving signals from the MEASAT-3 satellite, which is geostationary at $91.5^{\circ} \mathrm{E}$; at $11.096 \mathrm{GHz}$. Serdang Selangor is the southern-most state of the Kuala Lumpur region of Malaysia. Data analysis was conducted in two ways. Firstly, by performing statistical analysis on rain attenuation prediction models; and secondly, by making a comparison between measured data and the existing rain attenuation prediction model. Of all, the ITU-R model gave the lowest real mean square value of (2.2) for the three chosen states in Malaysia.
\end{abstract}

Keyword: Radio wave propagation; Rain attenuation; Satellite communication 